\title{
The stimulus-response overshadowing phenomenon with VI versus FI schedules of reinforcement
}

\author{
ROGER M. TARPY and JEAN E. ROBERTS \\ Bucknell University, Lewisburg, Pennsylvania \\ and \\ STEPHEN E. G. LEA and MARIE MIDGLEY \\ University of Exeter, Exeter, England
}

\begin{abstract}
Previous research has shown that response rates on a variable interval (VI) schedule of reinforcement decrease if a brief response-produced signal is given prior to reward. One explanation is that the signal overshadows the response because it is a better predictor of reinforcement. The S-R overshadowing effect does not occur with variable ratio (VR) schedules, however. Tarpy, Lea, and Midgley (1983) explained this fact by suggesting that the signal functions to enhance the salience of the temporal interval offset on the VI schedule (a characteristic not possessed by VR schedules), which then overshadows the response. In this experiment, the salience of the temporal interval was enhanced in another way: signaled or unsignaled reward was provided to rats responding on either a VI or fixed interval (FI) reward schedule. As predicted, rates were lowest for animals receiving signaled reinforcement on an FI schedule and highest for those receiving unsignaled reinforcement on a VI schedule.
\end{abstract}

It is clear that overshadowing has become one of the most important concepts in Pavlovian theory in the last decade, primarily because it has helped to focus our thinking about stimulus learning on the information value of a conditioned stimulus. Overshadowing is not limited to just stimuli, however. In two recent independent studies, Pearce and Hall (1978) and St. Claire-Smith (1979a) demonstrated that a stimulus may overshadow a response as well. In their experiments, rats were permitted to obtain food on a variable-interval (VI) schedule of reward. For the experimental subjects, reward was preceded by a brief light flash. Control subjects received an equal number of light flashes delivered randomly with respect to reward. The results showed that the subjects for which the reward was signaled pressed at a lower rate than the control subjects that received the random light presentations (stimulus-response, or S-R, overshadowing effect). Pearce and Hall and St. Claire-Smith argued that these results demonstrated a form of overshadowing: since the light was a good predictor of reward (food always followed it), but the response was a relatively poor predictor (only occasionally did a response yield reinforcement), the

These data were reported at the Psychonomic Society convention in Minneapolis, 1982. Reprints may be obtained from Roger M. Tarpy, Department of Psychology, Bucknell University, Lewisburg, PA 17837, or Stephen E. G. Lea, Department of Psychology, University of Exeter, Exeter EX4 4QG, England. more predictive or valid element (light) overshadowed the less predictive element (response), making the response association weaker. These experiments are important because they are among the first to suggest that the principles of stimulus learning apply to response learning as well (see also Dickinson, 1980; Fowler, Goodman, \& DeVito, 1977; Garrud, Goodall, \& Mackintosh, 1981; Hall, 1982; Hall, Channell, \& Pearce, 1981; Lattal \& Ziegler, 1982; Mackintosh \& Dickinson, 1979; Shettleworth, 1981; St. ClaireSmith, 1979b; Tarpy, 1982; Williams, 1975, 1978; Williams \& Heyneman, 1982).

In a recent paper, Tarpy, Lea, and Midgley (1983) examined some of the critical aspects of this phenomenon, in particular whether a poor response-reward correlation is sufficient to produce the effect. If a discrepancy between the stimulus-reward correlation and the response-reward correlation is, indeed, critical to the phenomenon, then overshadowing should be apparent under a variable ratio (VR) schedule of signaled reward just as it is under a VI schedule, because both schedules involve a relatively higher S-US than R-US correlation. Having replicated the S-R overshadowing effect (in Experiment 2), Tarpy et al. went on to show (in Experiments 3 and 4) that the effect does not occur when animals are trained on a VR schedule. Any theory that views the rate discrepancy under a VI schedule as an instance of overshadowing has severe difficulties explaining the absence of a difference under a VR schedule. 
Tarpy et al. attempted to explain their results within an information theory framework in the following way. Let us assume that the rate difference under VI schedules is a result of overshadowing. If, at the same time, we acknowledge that the differential correlation between food and the two explicit predictors (light and response), which exists under VR schedules just as under VI schedules, does not produce this rate difference, then it follows that some other predictive element is responsible for the overshadowing effect. The principal difference between interval and ratio schedules is that in the former there are two elements that must occur before reward is delivered, namely the expiration of the temporal interval and the response. On ratio schedules, in contrast, expiration of time is irrelevant to reward; only one event must occur for reward delivery, namely the execution of the appropriate number of responses. Therefore, since overshadowing, by definition, involves competition between two predictive elements, Tarpy et al. argued that the S-R overshadowing effect reflected competition between the temporal interval offset and the response; such an interval plays no role in VR schedules, a fact that accounts for the VI-VR discrepancy.

This line of argument accounts for the VI-VR difference, but it does not, of course, explain why the light stimulus affects the response rate on a VI schedule. To account for that fact, Tarpy et al. suggested that the light acts to enhance the salience of the temporal interval offset. If the light stimulus does operate by enhancing the salience of the interval offset, then it is reasonable to assume that similar rate discrepancies would be found if the offset were enhanced by some means other than the light. One way to highlight the boundaries of a temporal interval is to make them fixed. This is precisely the rationale for the present experiment. In addition to the normal signaled versus unsignaled VI condition, two additional groups of subjects were used. These received signaled or unsignaled reward under a fixed interval (FI) schedule. We predicted that overshadowing would occur (evidenced by a lower rate of responding) if the temporal interval offset were made salient by either means, that is, by having the interval boundaries fixed or by presenting the light cue prior to reward. In addition, we predicted that when both were present, that is, in the FI-signaled reward condition, the degree of overshadowing would be greater than when only one was present.

\section{METHOD}

\section{Subjects}

The subjects were 16 male and 16 female Long-Evans rats purchased from Charles River Breeding Labs and weighing between 191 and $290 \mathrm{~g}$ at the beginning of the experiment. They were given free access to water throughout the experiment, but were deprived of food $24 \mathrm{~h}$ before the first session. Thereafter, following each session, they were fed enough food so that they remained at about $85 \%$ of their preexperimental weights. All subjects were housed individually in standard wire mesh cages in a colony room in which the lights came on for $12 \mathrm{~h}$ at 8:00 a.m. All training sessions were conducted at around 11:00 a.m.

\section{Apparatus}

Eight identical lever boxes, manufactured by Coulbourn Instruments, were used. Each measured $30 \mathrm{~cm}$ long $\times 25.5 \mathrm{~cm}$ wide $\times 29 \mathrm{~cm}$ high and was placed in a larger sound-attenuating chamber equipped with a ventilating fan. A response lever was located in the center of the front wall $12 \mathrm{~cm}$ above the grid floor. A Coulbourn pellet trough, the opening measuring $3.5 \times 3.5 \mathrm{~cm}$, was situated $5 \mathrm{~cm}$ below the lever. A unit containing three jewel lights was located $3.5 \mathrm{~cm}$ above the response lever; the central yellow lamp was used as the signal. A houselight was centered at the top of the intelligence panel near the ceiling. Reinforcers consisted of 45-mg Noyes pellets (P. J. Noyes Co.). Computer control and recording equipment were located in an adjacent room.

\section{Procedure}

During the 2 days following deprivation, the rats were given pretraining in which food pellets were delivered every $60 \mathrm{sec}$ and all leverpresses were reinforced. A few rats were also hand shaped during this phase. All sessions were $30 \mathrm{~min}$ in duration, during which a houselight remained on.

During the 16 training sessions, all reinforcers were delivered $0.5 \mathrm{sec}$ after the reinforced response. For the correlated (C) rats, this delay was filled with a flash of the yellow light. Each $C$ rat had a yoked partner of the same sex which was tested in an experimental chamber that was neither horizontally nor vertically adjacent. Whenever a rat in a correlated group earned a signaled reward, an identical light flash was delivered to its yoked partner. Thus, rats in the random $(R)$ condition received the same delay of reward and the same number of light stimuli as their "executive" partners, but the flashes were not correlated with food delivery. The subjects also differed according to the schedule of reward used. Half the subject pairs in the $C$ and $R$ condition were run under a VI reward schedule; the other half were given an FI schedule. Thus, there were four basic conditions, each containing four male and four female rats: FI reward with a food-correlated signal (FI-C), FI reward with a random signal (FI-R), VI reward with a correlated cue (VI-C), and VI reward with a random signal (VI-R). On the 1st day of training, FI-.25 and VI-.25 (range $=5$ to $25 \mathrm{sec}$ ) schedules were used; on the 2nd day, FI-.5 and VI-.5 (range $=5$ to $55 \mathrm{sec}$ ) were used; and for the remaining 14 days of the experiment, FI-1 or VI-1 min schedules (range $=5$ to $115 \mathrm{sec}$ ) were used.

\section{RESULTS}

Figure 1 shows the mean response rates for each of the four groups across the 14 sessions during which the FI-1 and VI-1 schedules were in effect. For all sessions except the first, Group FI-C responded at the lowest rate and Group VI-R at the highest rate, with the other two groups responding at intermediate levels. The trends evident in Figure 1 were confirmed to be statistically significant. An analysis of variance showed significant effects due to schedule $[F(1,24)=$ $4.3, p<.05]$ and session $[F(13,312)=33.4, p<.0001]$ and the significant interaction of session $\times$ schedule $[F(13,312)=4.0, p<.0001]$. Surprisingly, the main effect due to signal (i.e., $C$ vs. $R$ ) was not significant $[F(1,24)=2.5, p=.12]$. However, an unexpectedly large effect due to $\operatorname{sex}[F(1,24)=11.7, p<.005]$ caused 


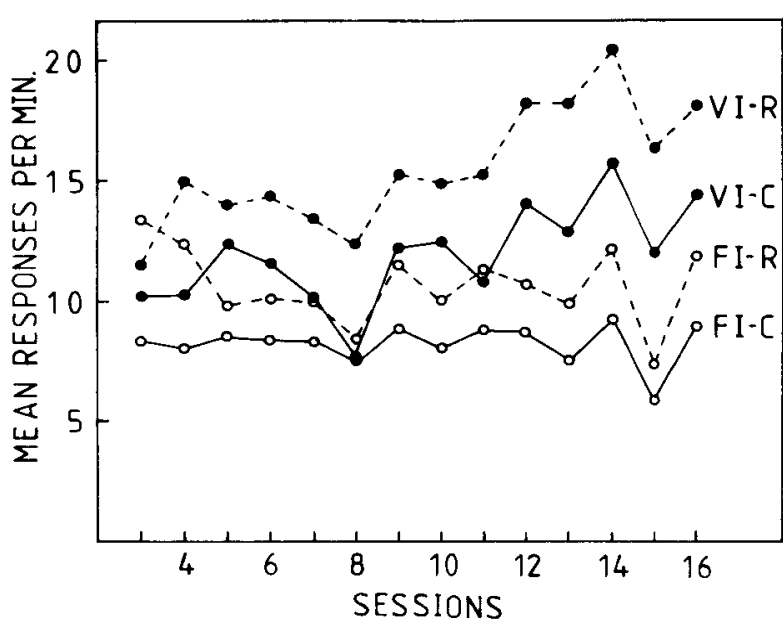

Figure 1. Mean lever responses per minute as a function of the 16 training sessions.

the data to have unusually large within-group variance. Accordingly, we transformed the data to logs to reduce this variability. An analysis of variance on these data yielded a significant effect due to the signal $[F(1,24)=4.2, p<.05]$.

There was no significant difference among groups in the number of reinforcers obtained per session, indicating that the difference in response rates did not affect the number of reinforcers collected.

The number of responses emitted during the delay interval was significantly higher in the $\mathbf{R}$ groups than in the $C$ groups $[F(1,24)=15.9, p<.001]$, suggesting that responding was suppressed in Group $C$ by the food-correlated light (see Lattal \& Ziegler, 1982). This finding, however, cannot account for the $C$ vs. $R$ difference. The mean total number of responses occurring during the lights over the 30 -min sessions was only $1.28,4.75,1.06$, and 3.76 for Groups VI-C, VI-R, FI-C, and FI-R respectively. Correction of the original overall data by subtraction of these values altered neither the general pattern nor the statistical significance of the results described above.

It could be argued that the rate difference between FI-R and VI-R was not due to the fact that the temporal interval was fixed in the former group, as we are claiming, but rather that the FI-R rats, inadvertently, received correlated light cues. This is, indeed, a possibility: the session for a given yoked pair of FI subjects (i.e., FI-C and FI-R) began at the same time; since only a few reinforcers are missed each session, the lights for the FI-R subjects could have occurred more or less just prior to their rewards. This hypothesis does not appear to be very credible, however, because it relies entirely on the close synchrony in reinforcement delivery for members of an FI-C and FI-R pair. Such synchrony is not suggested by the data. To illustrate, we analyzed the degree of synchrony during the last session. None of the subjects earned the maximum number of reinforcers possible; all "wasted" some time. Moreover, the amount of time wasted was typically different for the two members of each yoked pair. We estimated the numbers of seconds wasted for each animal by multiplying the number of reinforcers missed by $60 \mathrm{sec}$ (the actual time wasted may have been as much as $59 \mathrm{sec}$ less than this figure, depending upon when the session ended relative to the last obtained reward). Then we calculated the mean seconds wasted per reinforcer and the absolute difference in this figure for each yoked pair. The overall mean of the absolute differences in seconds wasted per reinforcer for all eight pairs of FI rats was $5.1 \mathrm{sec}$. If we make the conservative assumption that the wasted time was evenly distributed over all the earned reinforcers, then, on the average, the reward delivery for a given FI-C and FI-R pair was out of synchrony by $5.1 \mathrm{sec}$ on the first reward delivery, by 10.2 sec for the second reward, and so on. Maximum asynchrony would thus be reached after fewer than six reinforcers (i.e., after about $6 \mathrm{~min}$, or $20 \%$ of the session). Of course, there are other assumptions about the distribution of wasted time that could be made, but none is a priori more favorable than the one we made. Moreover, our subjective impression when collecting these data confirmed that the pattern of responding was asynchronous: It seemed as if a cluster of rewards was earned when the session first began (we can hear the click of the pulse formers that drive the feeders), but that rewards were delivered in quite an asynchronous fashion after several minutes. In summary, the reduced rate for FI-R subjects relative to VI-R rats does not appear to have been caused by light cues inadvertently occurring just prior to reward.

\section{DISCUSSION}

The results reported here replicate the S-R overshadowing effect and show further that the effect occurs with FI as well as VI schedules of reinforcement. This latter finding is not surprising, since FI and VI schedules are similar contingency arrangements. What is more interesting and critical for a theoretical understanding of the S-R overshadowing effect, however, is the overall FI-VI rate difference. That FI subjects responded at a lower rate than VI subjects agrees with reports in the literature. Mandell (1980), for example, used multiple FI,VI (Experiment 1) or multiple FI, random interval (Experiment 2) schedules in which the average rate of reinforcement was equal in the two components. Three of four birds in Experiment 1 and two of three birds in Experiment 2 responded at considerably lower rates in the FI component. Experiments employing a concurrent chain procedure with terminal links being either FI or VI (or mixed interval) but with equal average rate of reinforcement also have found 
lower rates in the FI terminal link (e.g., Davison, 1969, with three out of five birds; Davison, 1972, with five out of six birds; Herrnstein, 1964, with four out of four birds). In the present context, these results indicate that the degree of temporal control is important to the S-R overshadowing phenomenon. This would appear to support the argument advanced by Tarpy et al. (1983): the response association is overshadowed not by the light per se, but by the temporal interval offset whose salience is enhanced by the light. Temporal control is certainly evident under FI schedules. For example, it is well known that the regularity of the FI interval results in scalloping (see Dews, 1970), postreinforcement pausing (Ferster \& Skinner, 1957; Kendall, 1972), and "break and run" patterns (Schneider, 1969). Moreover, if further measures are taken to enhance the salience of the temporal interval, such as providing a cue that functions as a "clock," then temporal control is increased even more (see Donahoe, 1970; Kendall, 1972; Segal, 1962).

It is our assumption that temporal control also occurs on VI schedules, albeit less strongly than on FI schedules. Indeed, the extent of temporal control ought to be an inverse function of the consistency of the interval. And although subjects may have difficulty learning about the specific interval values employed, they certainly learn that the expiration of time is critical for reward. There are data to support this contention. For example, Catania and Reynolds (1968) showed a small, but reliable, scallop effect with VI schedules. Similar effects have been shown by other investigators (Harzem, Lowe, \& PriddleHigson, 1978; Leslie, 1981). In the Leslie experiment, acceleration of response rate late in the interval was eliminated only when the momentary probability of reward was zero, and the postreinforcement pause persisted even when the probability of reward in the early part of the interval was 1.0.

Although the difference in rate maintained by FI and VI schedules is entirely consistent with the hypothesis expressed above, we must add a word of caution. This difference could occur for reasons other than overshadowing by the temporal interval offset. For instance, the unique contingency created by an FI schedule that produces scalloping and long postreinforcement pauses could very well be the cause of an overall rate difference. Future research is required to clarify this issue.

If the $S-R$ overshadowing effect is due to competition between the interval offset and the response, then manipulation of the salience of the offset should produce systematic differences in the degree of overshadowing, that is, in the $C$ vs. $R$ rate differential. The results of this experiment, coupled with those in the literature on, for example, FI clocks, provides evidence for just such a belief. In particular, either manipulation-making the interval fixed or signaling the reward-produced a lower response rate, and both manipulations combined produced the lowest rate of all.

A number of other possible theories that could explain the $C$ versus $R$ difference have been offered (see Roberts, Tarpy, \& Lea, 1984) but none seems to be consistent with all the facts. And there is already some evidence suggesting that overshadowing, even by the temporal interval, may not accurately reflect the essential process underlying the $C$ versus $R$ difference (see Roberts et al., 1984). Although the present experiment does not provide any means for resolving these issues, it does offer evidence that, at the very least, the temporal interval plays an important part in the phenomenon: Not only is the temporal interval apparently essential for producing the S-R overshadowing effect (Tarpy et al., 1983), but its salience is an important determinant of the degree of the rate difference (present study).

\section{REFERENCES}

Catania, A. C., \& Reynolds, G. S. (1968). A quantitative analysis of the responding maintained by interval schedules of reinforcement. Journal of the Experimental Analysis of Behavior, 11, 327-383.

Davison, M. C. (1969). Preference for mixed-interval versus fixedinterval schedules. Journal of the Experimental Analysis of Behavior, 12, 247-252.

Davison, M. C. (1972). Preference for mixed-interval versus fixed-interval schedules: Number of component intervals. Journal of the Experimental A nalysis of Behavior, 17, 169-176.

Dews, P. B. (1970). The theory of fixed-interval responding. In W. N. Schoenfeld (Ed.), The theory of reinforcement schedules. New York: Appleton-Century-Crofts.

Dickinson, A. (1980). Contemporary animal learning theory. Cambridge: Cambridge University Press.

Donahoe, J. W. (1970). Stimulus control within response sequences. In J. H. Reynierse (Ed.), Current issues in animal learning: A colloquium. Lincoln: University of Nebraska Press.

Ferster, C. B., \& Skinner, B. F. (1957). Schedules of reinforcement. New York: Appleton-Century-Crofts.

Fowlen, H., Goodman, J. H., \& DiVito, P. L. (1977). Acrossreinforcement blocking effects in a mediational test of the CS's general signaling property. Learning and Motivation, 8, 507-519.

Garrud, P., Goodali, G., \& Mackintosh, N. J. (1981). Overshadowing of a stimulus-reinforcer association by an instrumental response. Quarterly Journal of Experimental Psychology, 33B, 123-135.

HaLL, G. (1982). Effects of a brief stimulus accompanying reinforcement on instrumental responding in pigeons. Learning and Motivation, 13, 26-43.

Hall, G., Channell, S., \& Pearce, J. M. (1981). The effects of a signal for free or for earned reward: Implications for the role of response-reinforcer associations in instrumental performance. Quarterly Journal of Experimental Psychology, 33B, 95-107.

Harzem, P., Lowe, C. F., \& Priddle-Higson, P. J. (1978). Inhibiting function of reinforcement: Magnitude effects on variable-interval schedules. Journal of the Experimental Analysis of Behavior, 30, 1-10.

Herrnstein, R. J. (1964). Aperiodicity as a factor in choice. Journal of the Experimental Analysis of Behavior, 7, 179-182.

KENDALL, S. B. (1972). Some effects of response-dependent clock stimuli in a fixed-interval schedule. Journal of the Experimental Analysis of Behavior, 17, 161-168.

LatTal, K. A., \& Ziegler, D. R. (1982). Briefly delayed rein- 
forcement: An interresponse time analysis. Journal of the Experimental Analysis of Behavior, 37, 407-416.

LESLIE, J. C. (1981). Effects of variations in local reinforcement rate on local response rate in variable interval schedules. Journal of the Experimental Analysis of Behavior, 35, 45-53.

Mackintosh, N. J., \& Dickinson, A. (1979). Instrumental (type II) conditioning. In A. Dickinson \& R. A. Boakes (Eds.), Mechanisms of learning and motivation: A memorial volume to Jerzy Konorski. Hillsdale, NJ: Erlbaum.

MANDELL, C. (1980). Response strength in multiple periodic and aperiodic schedules. Journal of the Experimental Analysis of Behavior, 33, 221-241.

Pearce, J. M., \& Hall, G. (1978). Overshadowing the instrumental conditioning of a lever-press response by a more valid predictor of the reinforcer. Journal of Experimental Psychology: Animal Behavior Processes, 4, 356-367.

Roberts, J. E., Tarpy, R. M., \& Lea, S. E. G. (1984). Stimulusresponse overshadowing: Effects of signaled reward in instrumental responding as measured by response rate and resistance to disruption. Journal of Experimental Psychology: Animal Behavior Processes, 10, 244-255.

St. Claire-Smith, R. (1979a). The overshadowing of instrumental conditioning by a stimulus that predicts reinforcement better than the response. Animal Learning \& Behavior, 7, 224-228.

St. Craire-Smith, R. (1979b). The overshadowing and blocking of punishment. Quarterly Journal of Experimental Psychology, 31, 51-61.
Schneider, B. A. (1969). A two-state analysis of fixed-interval responding in the pigeon. Journal of the Experimental Analysis of Behavior, 12, 677-687.

Segal, E. F. (1962). Exteroceptive control of fixed-interval responding. Journal of the Experimental Analysis of Behavior, $5,49-57$.

Shettleworth, S. J. (1981). Reinforcement and the organization of behavior in golden hamsters: Differential overshadowing of a CS by different responses. Quarterly Journal of Experimental Psychology, 33B, 241-255.

TARPY, R. M. (1982). Principles of animal learning and motivation. Glenview, IL: Scott, Foresman.

TARPY, R. M., LeA, S. E. G., \& Midgley, M. (1983). The role of response-US correlation in stimulus-response overshadowing. Quarterly Journal of Experimental Psychology, 35B, 53-65.

Williams, B. A. (1975). The blocking of reinforcement control. Journal of the Experimental Analysis of Behavior, 24, 215-227.

Williams, B. A. (1978). Information effects on the responsereinforcer association. Animal Learning \& Behavior, 6, 371-379.

Williams, B. A., \& Heyneman, N. (1982). Multiple determinants of "blocking" effects on operant behavior. Animal Learning \& Behavior, 10, 72-76.

(Manuscript received June 8, 1983; revision accepted for publication August 30, 1983.) 